\title{
MACC1 facilitates chemoresistance and cancer stem cell-like properties of colon cancer cells through the PI3K/AKT signaling pathway
}

\author{
JIANKAI WANG ${ }^{1}$, WENJUAN WANG $^{2}$, HONGYI CAI $^{1}$, BINBIN DU $^{3}$, LIJUAN ZHANG $^{1}$, \\ WEN MA $^{1}$, YONGGUO HU ${ }^{1}$, SHIFANG FENG ${ }^{1}$ and GUOYING MIAO ${ }^{1}$
}

${ }^{1}$ Department of Radiotherapy, Gansu Provincial Hospital; ${ }^{2}$ Department of Physical Examination Center, The Third People's
Hospital of Gansu; ${ }^{3}$ Department of Anorectal Surgery, Gansu Provincial Hospital, Lanzhou, Gansu 730050, P.R. China

Received November 24, 2016; Accepted August 16, 2017

DOI: 10.3892/mmr.2017.7721

\begin{abstract}
With regards to colon cancer, resistance to 5-fluorouracil (5-FU)-based chemotherapy and cancer stem cells (CSCs) are considered important factors underlying therapy failure. Metastasis-associated colon cancer 1 (MACC1) has been associated with poor prognosis and the promotion of metastasis within several types of cancer. However, the biological behavior of MACC1 in chemoresistance and CSC-like properties remains unclear. In the present study, various methods including gene knockdown, gene overexpression, western blotting, quantitative polymerase chain reaction and MTT assay, have been adopted. According to the results of the present study, MACC1 was depleted in two colon cancer cell lines resistant to 5-FU; subsequently, CSC-like properties and 5-FU sensitivity were investigated. Within 5-FU-resistant cells, cell death was facilitated by MACC1 knockdown. Furthermore, sphere formation and the expression levels of pluripotent markers, including cluster of differentiation (CD) 44, CD133 and Nanog were reduced due to MACC1 depletion. Additionally, it was indicated that the phosphoinositide 3-kinase/protein kinase B signaling pathway may be associated with 5-FU resistance and CSC-like properties via MACC1.
\end{abstract}

\section{Introduction}

Colon cancer is currently one of the most common malignant cancers of the gastrointestinal tract. The incidence of colon cancer has significantly increased in China within recent years (1). The associated resistance to cancer chemotherapy, in particular 5-fluorouracil (5-FU), is an increasing problem

Correspondence to: Dr Guoying Miao, Department of Radiotherapy, Gansu Provincial Hospital, 204 Donggangxi Road, Lanzhou, Gansu 730050, P.R. China

E-mail: 15339866618@163.com

Key words: metastasis-associated colon cancer 1, 5-fluorouracil, chemoresistance, cancer stem cells, colon cancer, protein kinase B within this line of treatment. Initially presented in 1997 , a small population of cells reported to have the ability to self-renew were identified as cancer stem cells (CSCs). Such cells pose a great challenge to cancer treatment due to associated chemoresistance (2).

Metastasis-associated colon cancer 1 (MACC1) was initially identified as a key regulator of the hepatocyte growth factor-tyrosine-protein kinase signaling pathway by genome-wide data analysis; MACC1 was also reported to be associated with colon cancer metastasis (3-5). Significant upregulation of MACC1 expression has been observed within malignant tissues including colon cancer of all stages, and liver and lung metastases, compared with in normal tissues and adenomas (3).

MACC1 promotes carcinogenesis, growth and metastasis of colorectal cancer $(6,7)$. Furthermore, MACC1 is associated with the promotion of tumor growth, invasion and metastasis in gastric cancer $(8,9)$, as well as poor prognosis in solid cancers $(10,11)$. As an easily detected biomarker in cancer, MACC1 may serve as a prognostic factor of remission following liver resection of colorectal cancer metastases (12). To the best of our knowledge, MACC1-associated regulation of cell chemoresistance, the development of CSC-like properties and the underlying mechanism have yet to be investigated.

Numerous mechanisms have been associated with the development of drug resistance. One of the major causes of multi-drug resistance (MDR) is the overexpression of the membrane-bound drug transporter protein P-glycoprotein (P-gp) $(13,14)$. P-gp is the protein product of the MDR gene ATP binding cassette subfamily B member 1 , and acts as an energy-dependent drug efflux pump that requires two molecules of adenosine 5'-triphosphate to remove a number of structurally unrelated chemotherapeutic drugs.

The present study detected MACC1 expression and examined its association with 5-FU resistance and CSC-like properties in colon cancer cells. Increased 5-FU sensitivity, reduced MDR protein 1 (MDR1) expression levels and reduced CSC-like properties were associated with MACC1 knockdown, which may be associated with inhibition of the phosphoinositide 3-kinase/protein kinase B (PI3K/AKT) signaling pathway. 


\section{Materials and methods}

Cell lines and reagents. SW620 and HCT116 cell lines were purchased from the Culture Collection of the Chinese Academy of Sciences (Shanghai, China). Cells were cultured in RPMI 1640 medium (Hyclone; GE Healthcare Life Sciences, Logan, UT, USA) supplemented with $10 \%$ fetal bovine serum (PAN Biotech $\mathrm{GmbH}$, Aidenbach, Germany) under a humidified $5 \% \mathrm{CO}_{2}$ atmosphere at $37^{\circ} \mathrm{C}$. In addition, 5-FU-resistant (5-FUR) cell lines SW620/5-FUR and HCT116/5-FUR were developed $(15,16)$. Briefly, SW620/5-FUR and HCT116/5-FUR cells were established by repeated subcultures in stepwise-increased concentrations of 5-FU (5, 10 and $20 \mu \mathrm{g} / \mathrm{ml}$; Sigma-Aldrich; Merck KGaA, Darmstadt Germany, USA) over 6 months. In the presence of $20 \mu \mathrm{g} / \mathrm{ml} 5-\mathrm{FU}$, exponential growth of SW620/5-FUR and HCT116/5-FUR cells was observed. Resistant cell lines were cultured under continuous 5-FU treatment $(20 \mu \mathrm{g} / \mathrm{ml})$ and were incubated in drug-free medium for 1 week prior to use. Primary antibodies against MACC1 (cat. no. ab106579) and MDR1 (cat. no. ab129450) were purchased from Abcam (Cambridge, UK). Primary antibodies against cluster of differentiation (CD) 44 (cat. no. 3570S), Nanog (cat. no. 4903) and AKT (cat. no. 4685) were purchased from Cell Signaling Technology, Inc. (Danvers, MA, USA). Primary antibodies against CD133 (cat. no. 18470-1-AP) and GAPDH (cat. no. 10494-1-AP) were obtained from Proteintech Group, Inc. (Chicago, IL, USA). Primary antibodies against phosphorylated AKT (p-AKT; Ser473; cat. no. 11054-1) were purchased from Signalway Antibody LLC (College Park, MD, USA). The PI3K inhibitor LY294002 was purchased from Selleck Chemicals (Houston, TX, USA). Cells were treated with $\mathrm{LY} 294002(20 \mu \mathrm{M})$ for $2 \mathrm{~h}$ at $37^{\circ} \mathrm{C}$.

Establishment of stably transfected cell lines. The short hairpin RNA (shRNA) sequence for MACC1 was: 5'-AAT TATATGCCAGGACAGCTT-3'. As a negative control (NC), a scrambled sequence was designed: 5'-AACAGTTATCTA TGCGACAGT-3'. Lenti-Easy Packaging Mix and Lentiviral Vector consist of shRNA (Shanghai GeneChem Co., Ltd., Shanghai, China) were transfected into $293 \mathrm{~T}$ cells. The cell supernatant was collected, concentrated by centrifugation $\left(4,000 \mathrm{x} \mathrm{g}, 37^{\circ} \mathrm{C}, 10 \mathrm{~min}\right)$ and purified. The lentivirus (LV)-MACC1-short hairpin RNA (shRNA)-green fluorescent protein (GFP) was obtained. The two colonic cancer cell lines were seeded on a 6 -well plate $\left(5 \times 10^{4}\right.$ cells/well) and cultured for $24 \mathrm{~h}$. Lentivirus (LV)-MACC1-short hairpin RNA (shRNA)-green fluorescent protein (GFP) was transduced into cells at a multiplicity of infection (MOI) of 60 using polybrene (10 $\mu \mathrm{g} / \mathrm{ml}$; Sigma-Aldrich; Merck KGaA) and Enhanced Infection Solution (Shanghai GeneChem Co., Ltd., Shanghai, China), named positive experimental group (shRNA group). Simultaneously, a non-target NC virus LV-NC-shRNA-GFP was transduced into cells as a control, noted as the vehicle group. After $12 \mathrm{~h}$, the medium was replaced with complete culture medium. Cells were cultured for $72 \mathrm{~h}$ in completed medium and observed under a fluorescence microscope for transduction efficiency. Then stably transduced cells were selected with $10 \mu \mathrm{g} / \mathrm{ml}$ puromycin. Reverse transcription-quantitative polymerase chain reaction
(RT-qPCR) and western blotting were performed to detect the inhibition rate of LV-mediated shRNA targeting MACC1.

For MACC1 overexpression, ectopic MACC1 coding sequence was amplified by PCR. The primer sequences were: Forward 5'-CCGCTCGAGATGCTAATCACTGAA AGAAAAC-3' and reverse 5'-CCGCTCGAGCTATACTTC CTCAGAAGTGGAGAAT-3'. Lenti-Easy Packaging Mix and Lentiviral Vector consist of MACC1 plasmid (Shanghai GeneChem Co., Ltd.,) were transfected into 293T cells. Cell supernatant were collected, concentrated and purified, Then the (LV)-MACC1-GFP) was obtained. Colonic cancer cells were seeded on a 6 -well plate $\left(5 \times 10^{4}\right.$ cells/well) and cultured for $24 \mathrm{~h}$. LV-MACC1-GFP was transduced into cells in the MAAC1 group at an MOI of 40 using polybrene $(10 \mu \mathrm{g} / \mathrm{ml}$; Sigma-Aldrich; Merck KGaA) and Enhanced Infection Solution (Shanghai GeneChem Co., Ltd.). Simultaneously, a non-target NC virus LV-NC-GFP was transduced into cells in the vehicle group. After $12 \mathrm{~h}$ the medium was replaced with complete culture medium, and stably transduced cells were selected with $10 \mu \mathrm{g} / \mathrm{ml}$ puromycin.

$R T-q P C R$. Cells were separated into three groups, including control group (no tranduction), vehicle group (transduction with LV-scrambled sequence-GFP) and shRNA group (transduction with LV-MACC1-shRNA-GFP). Total RNA was extracted from the cells following treatment for the indicated duration with RNAiso Plus (cat. no. 9108; Takara Biotechnology Co., Ltd., Dalian, China). Following genomic DNA elimination reaction and reverse-transcription reaction using PrimeScript ${ }^{\mathrm{TM}}$ RT reagent Kit with gDNA Eraser (Perfect Real Time; cat. no. RR047A; Takara Biotechnology Co., Ltd.) in a total volume of $20 \mu \mathrm{l}$ according to the manufacturer's protocol. First strand cDNA synthesis was conducted using 1,000 ng total RNA. Quantification of target and reference (GAPDH) genes was performed in triplicate using SYBR Premix Ex Taq II (Tli RNaseH Plus; cat. no. RR820A; Takara Biotechnology Co., Ltd.) on CFX96 Touch ${ }^{\text {TM }}$ Real-Time PCR Detection system (Bio-Rad Laboratories, Inc., Hercules, CA, USA). PCR thermocycling conditions is following: initial denaturation at $95^{\circ} \mathrm{C}$ $30 \mathrm{sec}$; 2 nd step PCR 40 cycles of $95^{\circ} \mathrm{C}$ for $5 \mathrm{sec}, 60^{\circ} \mathrm{C}$ for $30 \mathrm{sec}$ ) and dissociation. Following normalization to the GAPDH gene, expression levels for each target gene were calculated using the $2^{-\Delta \Delta C q}$ method (17). Primers used in RT-qPCR were as follow: MACC1, 5'-CCTTCGTGGTAATAATGCTTCC-3' forward and 5'-AGGGCTTCCATTGTATTGAGGT-3' reverse; CD44 forward, 5'-ATCATCTTGGCATCCCTCTTG-3' and reverse, 5'-CACCATTTCCTGAGACTTGCTG-3'; CD133 forward, 5'-ACA ATCCTGTTATGACAAGCCCA-3' and reverse, 5'-GGAAAGTCCTTGTAGACCCAGAAA-3'; Nanog forward, 5'-CCTATGCCTGTGATTTGTGGG-3' and reverse, 5'-TTGCCTTTGGGACTGGTGG-3'; Bmi-1 forward, 5'-AAG GAGGAGGTGAATGATAA-3' and reverse, 5'-ATCAATCTG GAAAGTATTAGG-3'; Oct-4 forward, 5'-GTGAGAGGCAAC CTGGAGAA-3' and reverse, 5'-GAACCACACTCGGACCAC AT-3'; GAPDH forward, 5'-ACCACAGTCCATGCCATCCA C-3' and reverse, 5'-TCCACCACCCTGTTGCTGTA-3'. Each experiment was performed in triplicate.

Western blotting. Cells were lysed in lysis buffer $(50 \mathrm{mM}$ Tris, $150 \mathrm{mM} \mathrm{NaCl}, 1 \%$ NP-40, 0.1\% SDS, $10 \mathrm{mM}$ EDTA, 
$1 \mathrm{mM}$ PMSF, and $0.5 \%$ sodium deoxycholate) containing phosphatase inhibitor. Following centrifuging at 13,201 x g at $4^{\circ} \mathrm{C}$ for $15 \mathrm{~min}$, the supernatants of lysates were collected for use. The proteins concentration was quantified by using the bicinchoninic assay protein assay kit (Beyotime Institute of Biotechnology, Shanghai, China). Lysates were denatured by heating. Equal amounts of protein samples $(20 \mu \mathrm{g})$ were loaded per well and separated by $10 \%$ SDS-PAGE, and were subsequently transferred electrophoretically onto polyvinylidene fluoride membranes. Following blocking with 5\% non-fat milk at room temperature for $1 \mathrm{~h}$, membranes were incubated with primary antibodies (all 1:1,000) at $4^{\circ} \mathrm{C}$ overnight and were then incubated with peroxidase-conjugated goat anti-rabbit immunoglobulin (Ig; cat. no. ZB-2301; OriGene Technologies, Inc., Beijing, China) or peroxidase-conjugated goat anti-mouse IgG (cat. no. ZB-2305; OriGene Technologies, Inc.; 1:5,000) for $1 \mathrm{~h}$ at room temperature. Specific immune complexes were detected using a chemiluminescence reagent (EMD Millipore, Billerica, MA, USA) with FUSION-FX7 advanced system (Vilber Lourmat, Marne La Vallée, France). Specific bands were quantified using FusionCapt Advance software (version 16.08a; Vilber Lourmat). Each experiment was performed in triplicate.

MTT assay. Cells (5.0x10 3 cells/well) were seeded in 96-well plates. Following treatment with a series of 5 -FU dosages $(5,10,20,40,80 \mu \mathrm{g} / \mathrm{ml})$ at $37^{\circ} \mathrm{C}$ for $48 \mathrm{~h}$, medium was removed and $50 \mu \mathrm{l}$ MTT (Sigma-Aldrich; Merck KGaA) was added to each well. Subsequently, cells were incubated at $37^{\circ} \mathrm{C}$ for $4 \mathrm{~h}$ in the dark. The reaction was terminated by the addition of $150 \mu$ l dimethyl sulfoxide (Sigma-Aldrich; Merck KGaA), and the absorbance of samples was measured at $570 \mathrm{~nm}$.

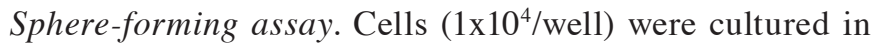
6 -well ultralow attachment surface dishes (Corning Inc., Corning, NY, USA). The cells were cultured in serum-free Dulbecco's modified Eagle's medium/F12 (Hyclone; GE Healthcare Life Sciences) supplemented with $20 \mathrm{ng} / \mathrm{ml} \mathrm{recom-}$ binant human epithelial growth factor (Peprotech EC Ltd. London, UK), $10 \mathrm{ng} / \mathrm{ml}$ human basic fibroblast growth factor (Peprotech EC Ltd.) and B27 (Gibco; Thermo Fisher Scientific Inc., Waltham, MA, USA). The number of spheres $>50 \mu \mathrm{m}^{3}$ was counted after 14 days of cell culture with an inverted microscope (CKX41; Olympus Corporation, Tokyo, Japan).

Statistical analysis. Data are expressed as the mean \pm standard deviation of three independent experiments. Data were analyzed by two-tailed unpaired Student's t-test between two groups and by one-way analysis of variance with Tukey's multiple comparison test among three groups. These analyses were performed using GraphPad Prism Software Version 6.0 (GraphPad Software, Inc., La Jolla, CA, USA).

\section{Results}

MACC1 expression is increased in 5-FUR colon cancer cells and is decreased by RNA interference. Human colon cancer cell lines SW620 and HCT116 were employed to generate SW620/5-FUR and HCT116/5-FUR cell lines via stepwise exposure to increasing doses of 5-FU.
Unexpectedly, compared with parental cells, the expression of MACC1 was significantly increased in 5-FUR cells (Fig. 1A). To determine the role of MACC1 in colon cancer cells, LV-MACC1-shRNA-GFP and LV-NC-shRNA-GFP were transduced into SW620/5-FUR and HCT116/5-FUR colon cancer cells. A total of $72 \mathrm{~h}$ post-transduction, cells with green fluorescence were observed under fluorescence microscopy (Fig. 1B). Cells were divided into three groups: Blank control cells (control group), NC cells (vehicle group) and MACC1-shRNA cells (shRNA group). Expression levels of MACC1 in transduced cells selected via puromycin were measured by RT-qPCR and western blot analysis. Compared with the control and vehicle groups, mRNA and protein expression levels of MACC1 were significantly downregulated in the shRNA group (Fig. 1C and D). Cells of the vehicle and shRNA groups were used for further analysis.

MACCl knockdown sensitizes colon cancer cells to 5-FU and reduces $M D R 1$ expression. To determine whether MACC1 promotes resistance to 5-FU in colon cancer cells, survival rate was detected in the vehicle and shRNA groups treated with a series of 5-FU dosages for $48 \mathrm{~h}$ via an MTT assay. Lower rates of survival were noted in the shRNA group, compared with in the vehicle group (Fig. 2A). Furthermore, MACC1 knockdown reduced the protein levels of MDR1 (Fig. 2B). Collectively, the results of the present study revealed that MACC1 promoted chemoresistance and MDR1 upregulation in SW620/5-FUR and HCT116/5-FUR cells.

MACC1 induces CSC-like properties of colon cancer cells. The effects of MACC1 on the CSC-like phenotype were investigated in SW620/5-FUR and HCT116/5-FUR cells. Cells in the shRNA group exhibited reduced sphere-forming capacity compared with cells in the vehicle group. Western blotting and RT-qPCR were employed to identify the genes responsible for the maintenance and regulation of CSC-like properties observed in SW620/5-FUR and HCT116/5-FUR cells. The results of the present study revealed a significant decrease in the expression levels of the pluripotent markers CD44, CD133, Bmi-1, Oct-4 and Nanog in the shRNA group compared with in the vehicle group (Fig. 3B and C). Collectively, these results suggested that MACC1 knockdown may reduce CSC-like properties in SW620/5-FUR and HCT116/5-FUR cells.

MACCl overexpression inhibits 5-FU sensitivity and promotes CSC-like properties in colon cancer cells. Western blot analysis was used to detect the expression levels of MACC1 in stably transduced cells. Compared with the control and vehicle groups, MACC1 protein levels were significantly upregulated in MACC1 group cells (Fig. 4A). To validate whether MACC1 overexpression promoted 5-FU resistance and stem cell-like properties in colon cancer cells, the survival rate was analyzed in the control, vehicle and $\mathrm{MACC1}$ groups treated with a series of 5-FU doses over $24 \mathrm{~h}$. The MACC1 group exhibited a higher rate of survival compared with the control and vehicle groups (Fig. 4B). Furthermore, MACC1 overexpression significantly increased MDR1 expression, and the expression of pluripotent markers CD44, CD133 and Nanog, compared with in the vehicle group (Fig. 4C). Collectively, these results demonstrated that MACC1 overexpression reduced 5-FU sensitivity, 

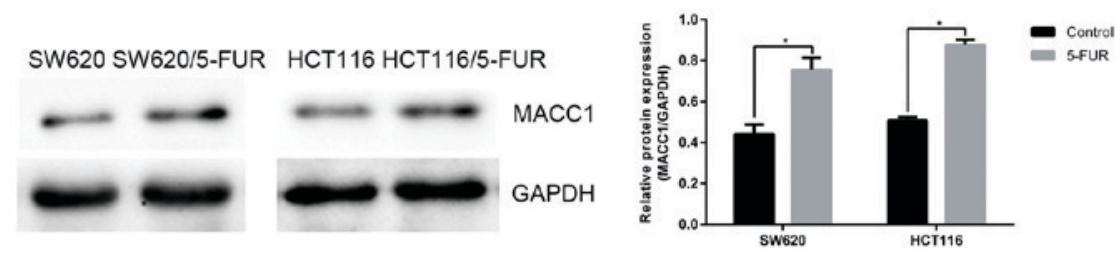

B
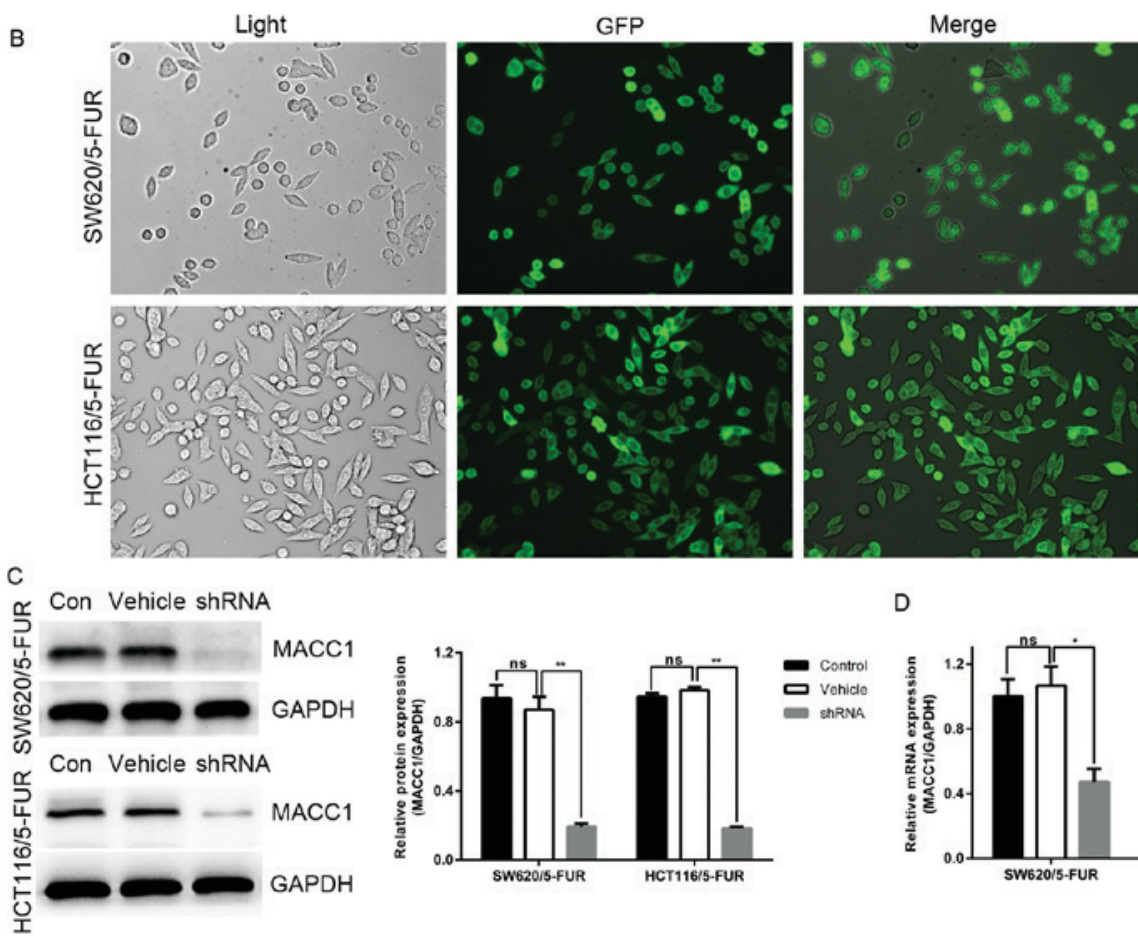

D

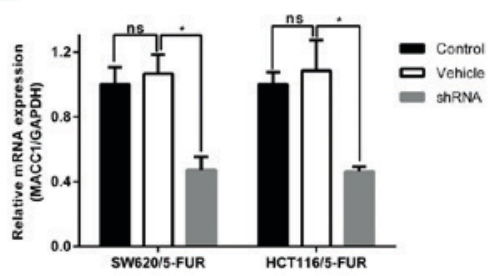

Figure 1. High expression of MACC1 in 5-FUR colon cancer cells and RNA interference-mediated MACC1 knockdown. (A) Western blot analysis of MACC1 expression in SW620 and HCT116 parental cells and 5-FUR cells. (B) SW620 and HCT116 cells transduced with the LV-MACC1-shRNA-GFP visible in normal light and under a fluorescent microscope. Merged images are presented (magnification, x200). (C) Bar graphs depict relative expression levels of MACC1 protein and (D) mRNA. LV-MACC1-shRNA exerted inhibitory effects on MACC1 expression, as identified by reverse transcription-quantitative polymerase chain reaction and western blot analysis. ${ }^{*} \mathrm{P}<0.05,{ }^{* *} \mathrm{P}<0.01$. 5-FUR, 5 -fluouracil-resistant; GFP, green fluorescent protein; LV, lentivirus; MACC1, metastasis-associated colon cancer 1; ns, not significant; shRNA, short hairpin RNA.

and induced CD44, CD133 and Nanog expression in SW620 and HCT116 cells.

MACC1 promotes chemoresistance and CSC-like properties via activation of the PI3K/AKT signaling pathway in colon cancer cells. Recent studies have revealed that AKT serves an important role in chemoresistance (18). Therefore, the phosphorylation levels of AKT (Ser473) in the control, vehicle and shRNA groups of HCT116/5-FUR cells were measured by western blotting. The results of the present study indicated that MACC1 knockdown decreased the protein expression levels of p-AKT (Fig. 5A). To determine the possible roles of PI3K/AKT in MACC1-induced chemoresistance and stem cell properties, LY294002 was applied; chemoresistance and sphere forming ability of HCT116/5-FUR cells were investigated. The results of the present study suggested that LY294002 significantly decreased 5-FU-associated resistance in HCT116/5-FUR cells (Fig. 5B). Protein expression levels of MDR1, CD133 and CD44 were decreased in response of HCT116/5-FUR cells to LY294002, compared with in the control group (Fig. 5C). Additionally, treatment with the PI3K inhibitor significantly reduced the sphere formation in HCT116/5-FUR cells (Fig. 5D). These results indicated that MACC1 may promote
5-FU-associated resistance and CSC-like properties of colon cancer cells via stimulation of the PI3K/AKT signaling pathway.

\section{Discussion}

The present study demonstrated that MACC1 may induce the development of 5-FU resistance and CSC-like properties in colon cancer cells. The results indicated that MACC1 was upregulated in 5-FUR colon cancer cells, whereas MACC1 knockdown promoted 5-FU sensitivity and reduced MDR1 protein expression. In addition to decreased sphere formation, MACC1 knockdown also reduced the expression levels of pluripotent markers CD44, CD133 and Nanog. Furthermore, the results of the present study suggested that the PI3K/AKT signaling pathway was involved in the regulatory effects of MACC1 in 5-FUR cells. To the best of our knowledge, the present study is the first to reveal that MACC1 promotes chemoresistance and CSC-like properties in colon cancer.

Patients with colon cancer that metastasizes to the liver are treated with conventional first-line treatments. Combinations of 5-FU, leucovorin and oxaliplatin or irinotecan are employed and have demonstrated efficiency in phase III trials (19). 
A

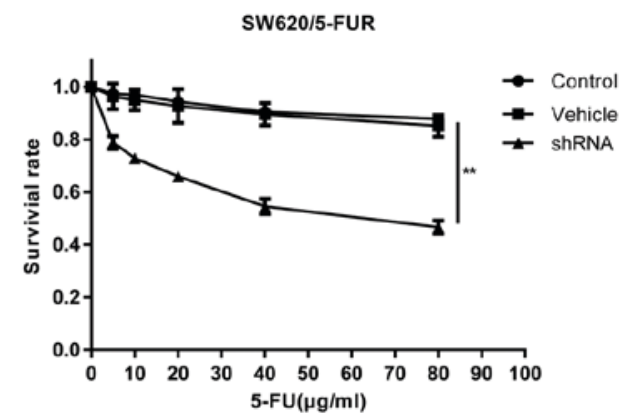

HCT116/5-FUR

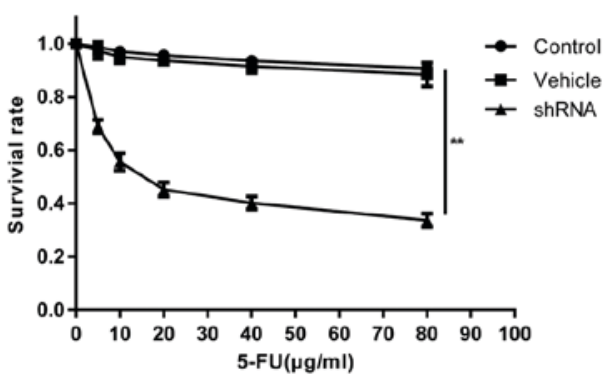

B
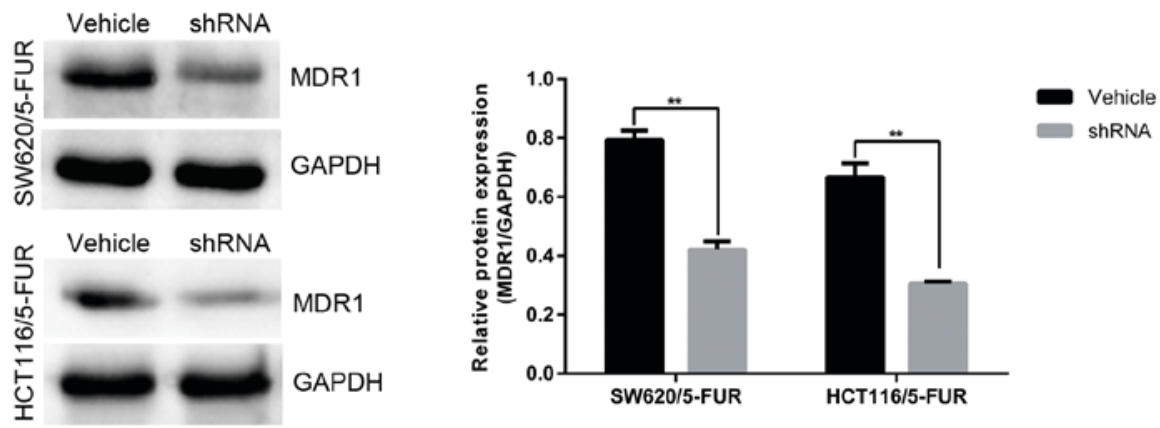

Figure 2. MACC1 knockdown sensitizes colon cancer cells to 5-FU and reduces MDR1 expression. (A) Vehicle group cells and shRNA group cells were cultured in the presence of 5-FU at the indicated concentrations for $24 \mathrm{~h}$, cell viability was determined using an MTT assay. (B) Protein levels of MDR1 were analyzed by western blotting in the vehicle and shRNA group cells. Bar graphs demonstrate the relative protein expression levels. ${ }^{* *} \mathrm{P}<0.01 .5$-FUR, 5-fluouracil-resistant; MACC1, metastasis-associated colon cancer 1; MDR1, multi-drug resistance protein 1; shRNA, short hairpin RNA.

A

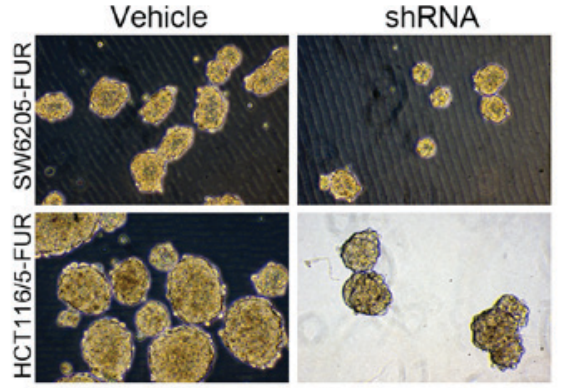

B

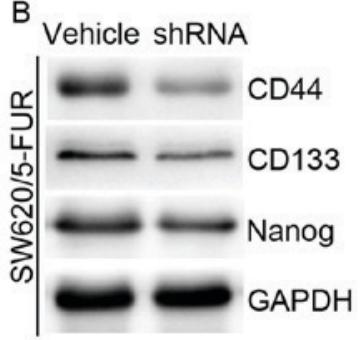

Vehicle shRNA

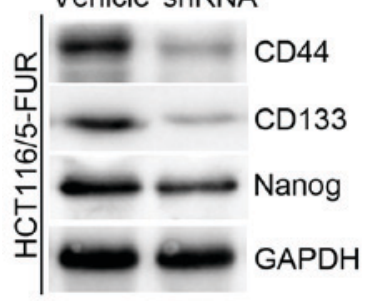

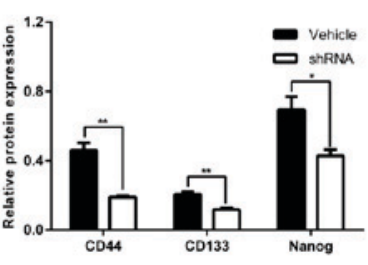
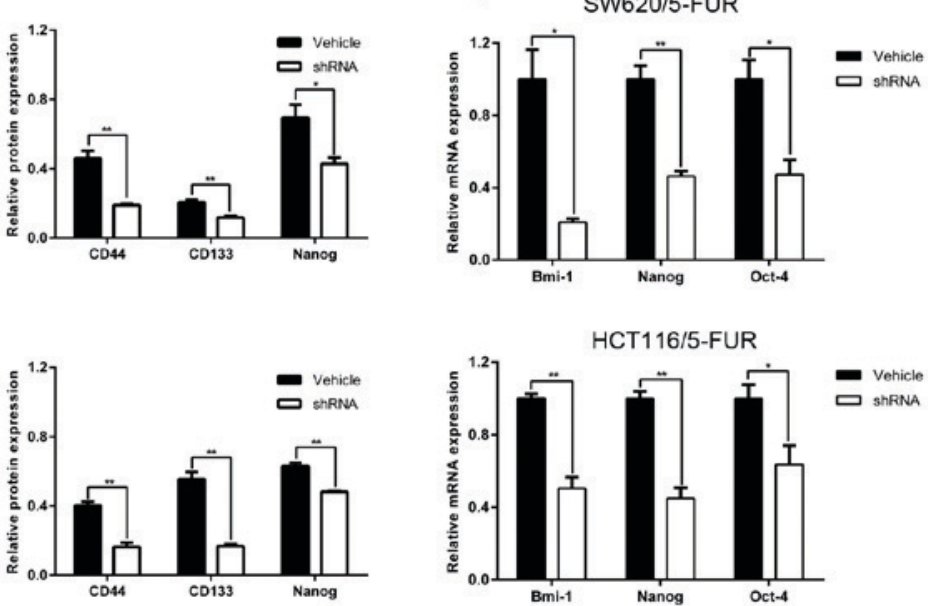
SW620/5-FUR

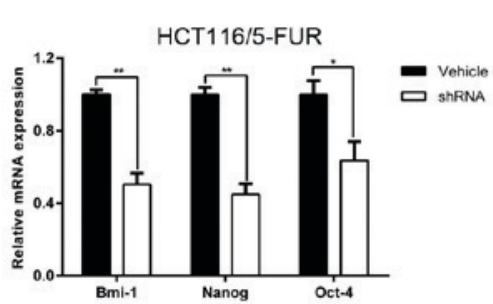

C

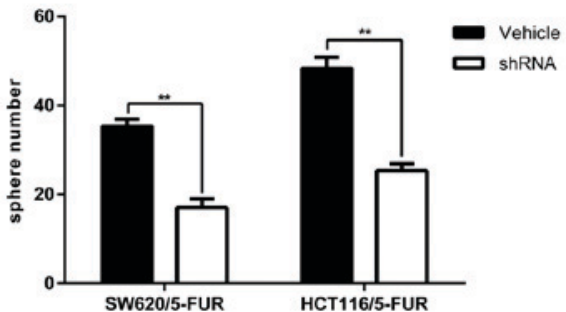

Figure 3. MACC1 knockdown reduces cancer stem cell-like properties of 5FUR colon cancer cells. (A) Sphere forming ability was evaluated in the vehicle group cells and shRNA group cells (magnification, x200). Bar graphs demonstrate the relative sphere numbers. (B) Protein levels of CD44, Nanog and CD133 were analyzed by western blotting in the vehicle group and shRNA group cells. Bar graphs demonstrate the relative protein expression levels. (C) mRNA levels of the aforementioned genes were analyzed by reverse transcription-quantitative polymerase chain reaction in the vehicle group and shRNA group cells. Bar graphs demonstrate the relative mRNA expression levels. ${ }^{*} \mathrm{P}<0.05,{ }^{* *} \mathrm{P}<0.01$. 5-FUR, 5-fluouracil-resistant; CD, cluster of differentiation; MACC1, metastasis-associated colon cancer 1 ; Oct-4, octamer-binding transcription factor 4; shRNA, short hairpin RNA. 


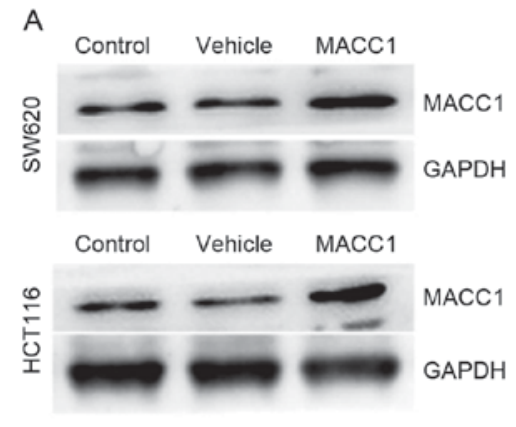

8

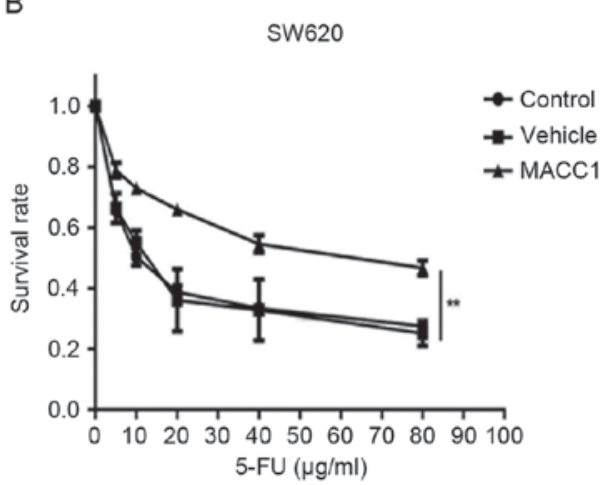

c

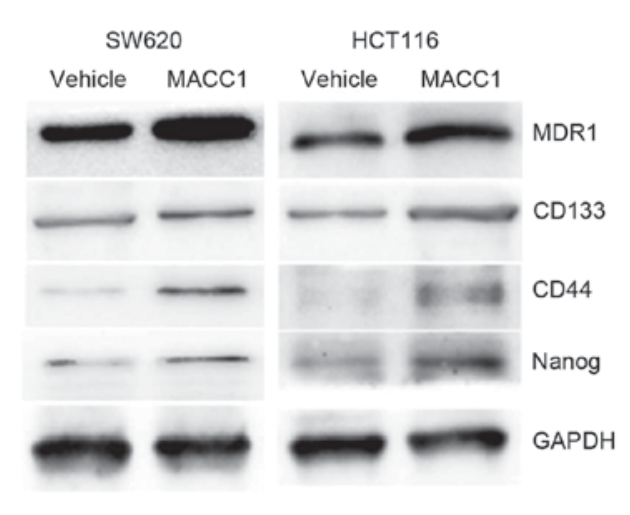

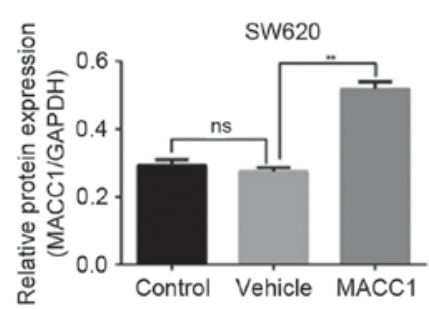

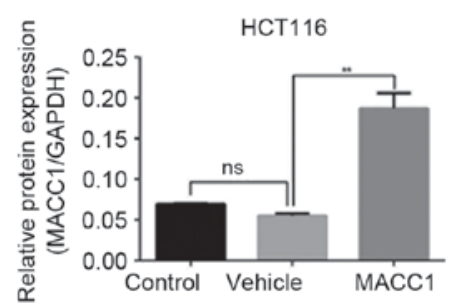

HCT116
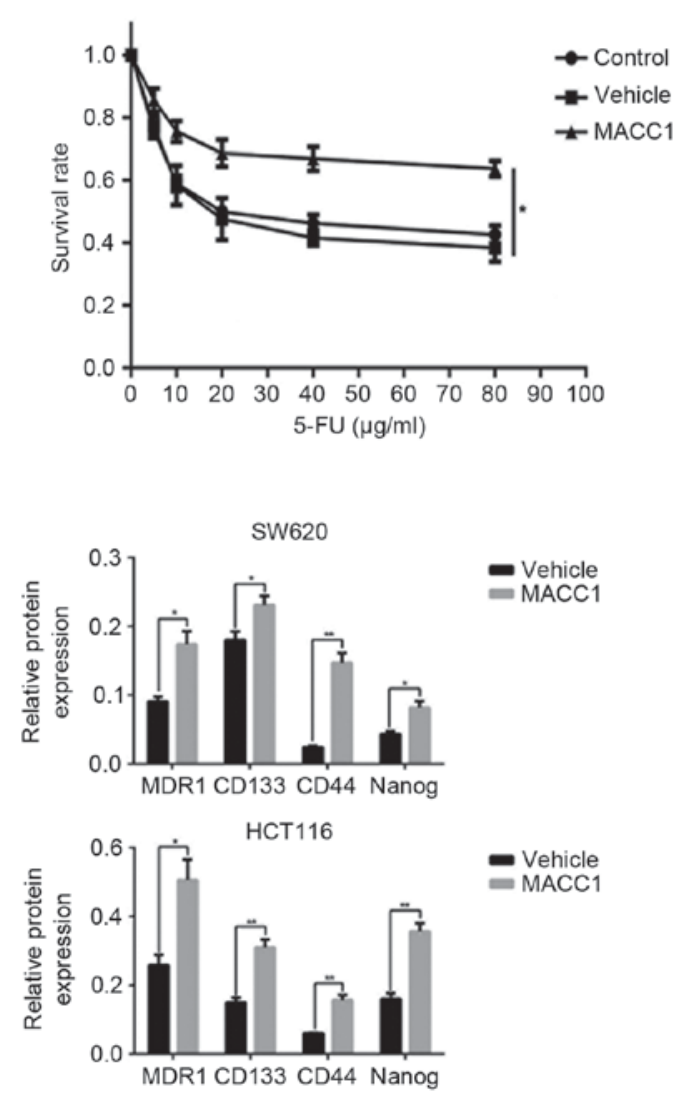

Figure 4. MACC1 overexpression inhibits 5-FU sensitivity and promotes cancer stem cell-like properties in colon cancer cells. Cells transduced with nothing comprised the control group, cells transduced with LV-negative control-GFP comprised the vehicle group and cells transduced with LV-MACC1-GFP comprised the MACC1 group. (A) Effects of MACC1 overexpression were identified by western blot analysis. Bar graphs demonstrate relative expression levels of MACC1 protein. ${ }^{* *} \mathrm{P}<0.01$ vs. vehicle (B) Cells of the control, vehicle and MACC1 groups were cultured in the presence of 5-FU at the indicated concentrations for $24 \mathrm{~h}$, cell viability was determined using an MTT assay. ${ }^{*} \mathrm{P}<0.05,{ }^{* *} \mathrm{P}<0.01$ vs. the vehicle (C) Protein levels of MDR1, CD133, Nanog and CD44 in the vehicle and MACC1 groups were analyzed by western blotting. Bar graphs indicate the relative protein expression levels. ${ }^{*} \mathrm{P}<0.05,{ }^{* *} \mathrm{P}<0.01 \mathrm{vs}$. vehicle. 5-FU, 5-fluorouracil; CD, cluster of differentiation; GFP, green fluorescent protein; LV, lentivirus; MACC1, metastasis-associated colon cancer 1; MDR1, multi-drug resistance protein 1; ns, not significant.

However, chemoresistance develops in almost all patients (2). Therefore, an improved understanding of drug-associated mechanisms of resistance is essential in exploring approaches for the prevention or reversal of drug resistance. MDR is a phenomenon that often accompanies drug treatment and is characterized by relapse or attenuation of drug efficacy; MDR is almost unavoidable in patients with colorectal cancer (CRC) receiving 5-FU-based chemotherapy. The MDR1 phenotype involves the development of resistance to numerous chemotherapeutics observed within cancer cells (20); the mechanisms responsible for MDR1 upregulation in CRC cells remain to be elucidated. In the present study, MACC1 knockdown promoted sensitivity to 5-FU and inhibited MDR1 protein expression in SW620/5-FUR and HCT116/5-FUR cells. MACC1 may therefore facilitate the development of chemoresistance in colon cancer.

CSCs are characterized by three unique properties: The capacity of self-renewal, the ability to exhibit all characteristics of the cancer cells of the parental tumor, and the expression of a distinctive set of surface biomarkers (21). Antitumor therapies may lead to a reduction of tumor mass yet fail to result in tumor regression due to the lack of CSC targeting. In the present study, MACC1 knockdown reduced sphere formation and the expression of pluripotent markers, including CD44, 
A

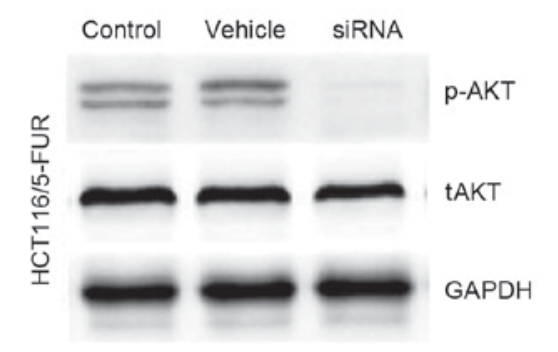

B
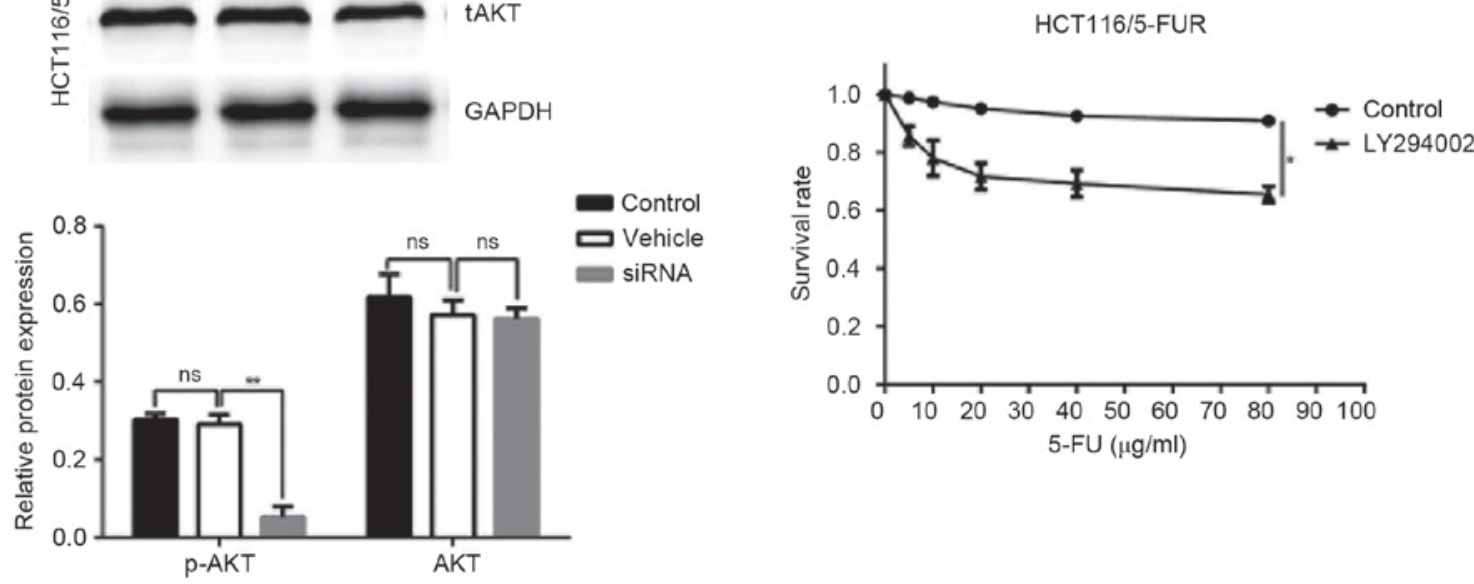

C

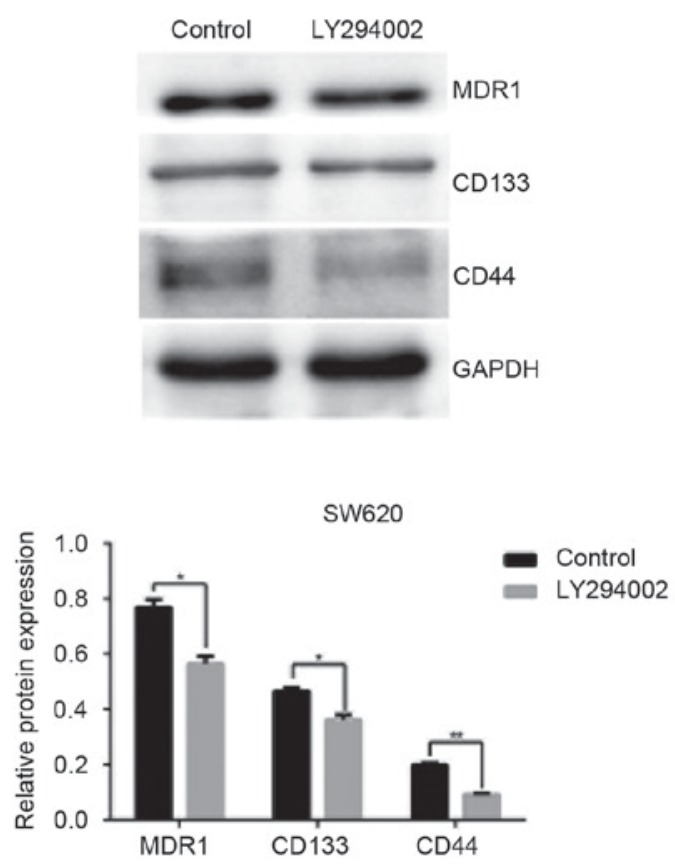

D
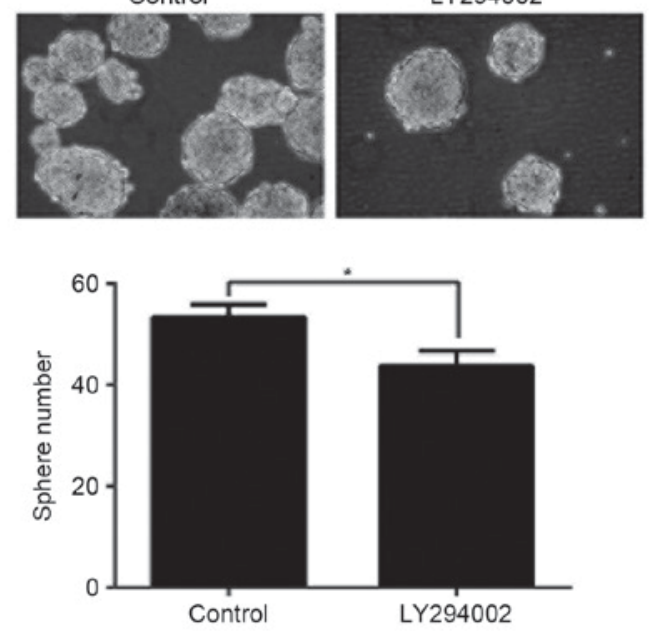

Figure 5. MACC1 promotes chemoresistance and cancer stem cell-like properties through the phosphoinositide 3-kinase/AKT pathway in 5FUR colon cancer cells. (A) Representative western blot image demonstrates the expression of tAKT and p-AKT in the control, vehicle and shRNA groups. Bar graphs indicate the relative protein expression levels. (B) Control group and LY294002 $(10 \mu \mathrm{M})$ group cells were cultured in the presence of 5-FU at the indicated concentrations for $24 \mathrm{~h}$, and the cell viability was determined using an MTT assay. (C) Protein levels of MDR1, CD133 and CD44 in the control and LY294002 (10 $\mu \mathrm{M})$ groups were analyzed by western blotting. (D) Sphere forming ability was evaluated in the control and LY294002 (10 $\mu$ M) group cells (magnification, x200). Bar graph demonstrate the relative sphere numbers. " $\mathrm{P}<0.05,{ }^{* *} \mathrm{P}<0.01 .5$-FU, 5 -fluorouracil; $\mathrm{CD}$, cluster of differentiation; AKT, protein kinase B; MACC1, MACC1, metastasis-associated colon cancer 1; MDR1, multi-drug resistance protein 1; ns, not significant; p-AKT, phosphorylated protein kinase B; shRNA, short hairpin RNA; tAKT, total protein kinase B.

CD133 and Nanog; MACC1 may serve as a potential target for the elimination of CSCs in colon cancer. Further research is required to determine the role of $\mathrm{MACC} 1$ in CSC-like properties and to understand the underlying mechanisms.

Recent studies revealed that PI3K/AKT serves an important role in chemoresistance $(18,22)$. Activation of the PI3K/AKT pathway contributes to numerous cancer therapy-associated resistance and is deemed a poor prognostic factor of cancer (23).
The results of the present study suggested that the protein expression levels of p-AKT were lower, indicating reduced activation of PI3K/AKT, in the MACC1-depleted colon cancer cells. Subsequent treatment of HCT116/5-FUR cells with LY294002 led to a decrease in cell survival rate and sphere formation compared with in untreated HCT116/5-FUR cells. These results indicated that the PI3K/AKT signaling pathway may serve a key role in 5-FU resistance and CSC-like 
properties via MACC1; however, this process requires further investigation.

\section{References}

1. Chen W, Zheng R, Zeng H, Zhang S and He J: Annual report on status of cancer in China, 2011. Chin J Cancer Res 27: 2-12, 2015.

2. Dallas NA, Xia L, Fan F, Gray MJ, Gaur P, van Buren G II, Samuel S, Kim MP, Lim SJ and Ellis LM: Chemoresistant colorectal cancer cells, the cancer stem cell phenotype, and increased sensitivity to insulin-like growth factor-I receptor inhibition. Cancer Res 69: 1951-1957, 2009.

3. Stein U, Walther W, Arlt F, Schwabe H, Smith J, Fichtner I, Birchmeier W and Schlag PM: MACC1, a newly identified key regulator of HGF-MET signaling, predicts colon cancer metastasis. Nat Med 15: 59-67, 2009.

4. Stein U, Smith J, Walther W and Arlt F: MACC1 controls Met: What a difference an Sp1 site makes. Cell Cycle 8: 2467-2469, 2009.

5. Stein U, Burock S, Herrmann P, Wendler I, Niederstrasser M, Wernecke KD and Schlag PM: Circulating MACC1 transcripts in colorectal cancer patient plasma predict metastasis and prognosis. PLoS One 7: e49249, 2012.

6. Zhen T, Dai S, Li H, Yang Y, Kang L, Shi H, Zhang F, Yang D, Cai S, He Y, et al: MACC1 promotes carcinogenesis of colorectal cancer via $\beta$-catenin signaling pathway. Oncotarget 5: 3756-3769, 2014.

7. Tang J, Chen J, Chen L, Tang J, Cui Z, Liu C and Wang Z: Metastasis associated in colon cancer 1 (MACC1) promotes growth and metastasis processes of colon cancer cells. Eur Rev Med Pharmacol Sci 20: 2825-2834, 2016.

8. Xie QP, Xiang C, Wang G, Lei KF and Wang Y: MACC1 upregulation promotes gastric cancer tumor cell metastasis and predicts a poor prognosis. J Zhejiang Univ Sci B 17: 361-366, 2016.

9. Yang T, He W, Cui F, Xia J, Zhou R, Wu Z, Zhao Y and Shi M: MACC1 mediates acetylcholine-induced invasion and migration by human gastric cancer cells. Oncotarget 7: 18085-18094, 2016.

10. Wang G, Fu Z and Li D: MACC1 overexpression and survival in solid tumors: A meta-analysis. Tumor Biol 36: 1055-1065, 2015.

11. Stein U: MACC1-a novel target for solid cancers. Expert Opin Ther Targets 17: 1039-1052, 2013.
12. Isella C,Mellano A, Galimi F, Petti C,Capussotti L, De Simone M, Bertotti A, Medico E and Muratore A: MACC1 mRNA levels predict cancer recurrence after resection of colorectal cancer liver metastases. Ann Surg 257: 1089-1095, 2013.

13. Yang X, Yang P, Shen J, Osaka E, Choy E, Cote G, Harmon D, Zhang Z, Mankin H, Hornicek FJ and Duan Z: Prevention of multidrug resistance (MDR) in osteosarcoma by NSC23925. Br J Cancer 110: 2896-2904, 2014.

14. Gottesman MM, Fojo T and Bates SE: Multidrug resistance in cancer: Role of ATP-dependent transporters. Nat Rev Cancer 2: 48-58, 2002.

15. Toden S, Okugawa Y, Jascur T, Wodarz D, Komarova NL, Buhrmann C, Shakibaei M, Boland CR and Goel A: Curcumin mediates chemosensitization to 5-fluorouracil through miRNA-induced suppression of epithelialto-mesenchymal transition in chemoresistant colorectal cancer. Carcinogenesis 36: 355-367, 2014.

16. Toden S, Tran HM, Tovar-Camargo OA, Okugawa Y and Goel A: Epigallocatechin-3-gallate targets cancer stem-like cells and enhances 5-fluorouracil chemosensitivity in colorectal cancer. Oncotarget 7: 16158-16171, 2016.

17. Livak KJ and Schmittgen TD: Analysis of relative gene expression data using real-time quantitative PCR and the 2(-Delta Delta C(T)) method. Methods 25: 402-408, 2001

18. Das D, Satapathy SR, Siddharth S, Nayak A and Kundu CN: NECTIN-4 increased the 5-FU resistance in colon cancer cells by inducing the PI3K-AKT cascade. Cancer Chemother Pharmacol 76: 471-479, 2015.

19. Lenz HJ: First-line combination treatment of colorectal cancer with hepatic metastases: Choosing a targeted agent. Cancer Treat Rev 34 (Suppl 2): S3-S7, 2008.

20. Amiri-Kordestani L, Basseville A, Kurdziel K, Fojo AT and Bates SE: Targeting MDR in breast and lung cancer: Discriminating its potential importance from the failure of drug resistance reversal studies. Drug Resist Updat 15: 50-61, 2012.

21. Maenhaut C, Dumont JE, Roger PP and van Staveren WC: Cancer stem cells: A reality, a myth, a fuzzy concept or a misnomer? An analysis. Carcinogenesis 31: 149-58, 2010.

22. Zhao M, Luo R, Liu Y, Gao L, Fu Z, Fu Q, Luo X, Chen Y, Deng X, Liang Z, et al: miR-3188 regulates nasopharyngeal carcinoma proliferation and chemosensitivity through a FOXO1-modulated positive feedback loop with mTOR-p-PI3K/AKT-c-JUN. Nat Commun 7: 11309, 2016.

23. LoPiccolo J, Blumenthal GM, Bernstein WB and Dennis PA: Targeting the PI3K/Akt/mTOR pathway: Effective combinations and clinical considerations. Drug Resist Updat 11: 32-50, 2008. 